\title{
Evaluación de la percepción de los alumnos al ruido exterior e interior en el campus de la Universidad Nacional Agraria La Molina
}

\author{
Perceptual evaluation of students to the exterior and interior noise in the campus of the \\ Universidad Nacional Agraria La Molina
}

Julia Carolina Rivadeneyra ${ }^{1}$ \& Luis Yoza $^{2}$

\begin{abstract}
Resumen
Se evaluó la percepción de los estudiantes frente al ruido interior en la Biblioteca Agrícola Nacional (BAN) y el Comedor Universitario y al ruido exterior de las áreas académicas de la Universidad Nacional Agraria La Molina (UNALM). Se aplicó encuestas, además se elaboraron mapas de ruido interno y externo de las áreas de estudio. La mayoría de estudiantes sienten molestia debido al ruido ambiental durante las horas de la mañana, sobre todo por el podado de los jardines durante horas de clase; asimismo, afirman que el área más ruidosa es aquella en la que se ubican las aulas del módulo azul. Los estudiantes que asisten con frecuencia a la BAN afirmaron sentir molestia y pérdida de la concentración por los altos niveles de ruido; en el caso del comedor universitario, también se genera molestia por el ruido interior. Se encontró que el género femenino es ligeramente más sensible al ruido que el género masculino. En los mapas de ruido realizados dentro de las áreas académicas de la UNALM, se evidenció que los niveles de ruido exceden la norma nacional para ruido exterior; en efecto, se registraron los valores más altos de ruido en áreas cercanas a las pistas, al comedor universitario y donde se ubican las aulas del módulo azul. Además, el ruido interior en la BAN y en el comedor universitario supera en 20 dB la recomendación de la Organización Mundial de la Salud.
\end{abstract}

Palabras clave: mapa de ruido; percepción; ruido ambiental; ruido interior.

\begin{abstract}
We evaluated the perception of students towards the indoor noise in the "Biblioteca Agrícola Nacional (BAN)" and the university dining hall, and outdoor noise in the academic areas. We used surveys and we also elaborated maps of outdoor and indoor noise within the study areas. Most students feel discomfort due to environmental noise during the morning hours, mainly because of the pruning of gardens during class hours, moreover they affirm that the noisiest area is where the blue module classrooms are located. Students who frequently assist to the BAN affirm that they feel uncomfortable and loss of concentration due to high noise levels; in the university dining hall students also feel discomfort caused by the indoor noise. It was found that the feminine gender is slightly more sensitive to noise than the masculine gender. In the noise maps developed within the academic area of the La Molina University, we found that the noise levels exceed the national standard for outdoor noise, the highest levels of noise were recorded in the areas near to roads, the university dining hall and in the area of the blue module classrooms. Furthermore, the indoor noise recorded in the BAN and the university dining hall exceeds in $20 \mathrm{~dB}$ the World Health Organization (WHO) recommendation.
\end{abstract}

Keywords: indoor noise; noise map; outdoor noise; perception.

\section{Introducción}

Uno de los problemas ambientales que se viene afrontando en las grandes ciudades es el ruido producido por las diferentes actividades de producción y recreación ya que las personas expuestas a altos niveles de ruido pueden desarrollar problemas de salud a nivel psicológicos y físicos (De Esteban, 2003).

La comunidad científica ha mostrado interés en la investigación de la contaminación acústica hace pocos años; en el Perú adquiere mayor énfasis a partir del año 1993 cuando se promulgó el Código del Medio Ambiente (Santos, 2007). Actualmente, se establecen los lineamientos respecto al ruido en la Ley General del Ambiente y se hace obligatorio el cumplimiento de los estándares de calidad ambiental.

Para lugares destinados a la enseñanza, la Organización Mundial de la Salud (OMS) ha recomendado valores máximos de niveles de ruido exterior e interior (OMS, 1999), ya que altos niveles de ruido conllevan a una disminución de la eficiencia en la labor desempañada,

\footnotetext{
${ }^{1}$ Investigadora asociada al laboratorio de Contaminación Acústica. Universidad Nacional Agraria La Molina, Lima, Perú.

${ }^{2}$ Facultad de Ciencias. Universidad Nacional Agraria La Molina, Lima, Perú. Email: lyoza@lamolina.edu.pe
} 
además de generar molestia y posibles problemas psicológicos (Basrur, 2000); es por eso que es imprescindible que las áreas destinados para labores estudiantiles cumplan con esta recomendación.

Si bien el área donde se ubica la Universidad Nacional Agraria La Molina no presenta altos niveles de ruido (Arellano, 2008), dentro de la misma existen puntos y horas estratégicas donde las propias actividades estudiantiles generan niveles de ruido que pueden causar efectos negativos sobre las personas expuestas; aunque estos niveles no provocan problemas físicos, si son la causa de la sensación de molestia, inconformidad y estrés (Piñon y Barcelo, 2005); además es de suma importancia conocer la percepción de los estudiantes respecto al ruido exterior e interior, así como la molestia que éste les puede generar y cómo interfiere en sus labores académicas.

A través de la presente investigación se pretende evaluar la percepción de los estudiantes al ruido en interiores y exteriores del campus de la Universidad Nacional Agraria la Molina (UNALM) y caracterizar el ruido interior y exterior en las áreas académicas de la misma.

\section{Materiales y métodos}

El área de estudio fue aquella en la cual se desarrollan las actividades académicas de la UNALM, mostrada en la Fig. 1; ubicada en el distrito de La Molina, provincia de Lima, Departamento Lima.

Para la evaluación de la percepción del ruido se elaboraron tres encuestas, las cuales tenían como público objetivo a los estudiantes de pregrado de la UNALM; el propósito de cada encuesta fue determinar el grado de molestia del ruido ambiental en el campus universitario, del ruido interior en la Biblioteca Agrícola Nacional y en el Comedor Universitario y poder diferenciar las opiniones respecto a género y lugar de procedencia.

El número de tamaño de muestra fue calculado según Steel y Torrie (1985) tomando como base un muestreo piloto de 50 encuestas y considerando la "sensibilidad al ruido" como la variable más significativa del estudio. Para comparar los resultados obtenidos en las encuestas con el ruido existente en el área de estudio, se elaboraron mapas de ruido ambiental en las áreas académicas de la UNALM y ruido interior de la Biblioteca Agrícola Nacional y del Comedor Universitario; la toma de datos se realizó con tres sonómetros clase 2 EXTECH 407780, debidamente calibrados y teniendo en cuenta las recomendaciones de la norma NTP/ISO 1996 - 2, 2008 para cada caso.

El monitoreo del ruido interior y exterior se realizó durante los meses de noviembre y diciembre del 2010 y enero del 2011 entre la 9 y las 14 horas, ya que es el horario en el cual se desarrollan la mayoría de actividades académicas de la UNALM; los datos fueron tomados en intervalos de 15 minutos con un tiempo de integración de 10 segundos y 5 repeticiones por cada estación de monitoreo; posteriormente, se determinó el nivel de presión sonora continua equivalente de cada punto evaluado.

Para la elaboración del Mapa de Ruido Ambiental se utilizó el "Software" Arc Gis 9.3 y su interpolador de datos "Ordinary Kriging" que dio como resultado un mapa con curvas isófonas en el área de evaluación.

\section{Resultados y discusión}

Percepción del ruido ambiental por los estudiantes.

De toda la población estudiantil encuestada, el $75 \%$ se considera medianamente sensible al ruido, el $17 \%$ se considera muy sensible y el $8 \%$ nada sensible, como se muestra en la Fig. 2. De otro lado, se encontró que el $54 \%$ de la muestra considera que el ruido dentro de la UNALM es un poco molesto, el $21 \%$ afirma que es molesto, otro $21 \%$ considera que el ruido no es molesto y menos del $4 \%$ no percibe ningún ruido molesto.

Según la percepción de los estudiantes, el ruido más molesto del campus universitario es el generado por las podadoras (44 \%), ya que son utilizadas durante el horario de actividades académicas, en segundo lugar se tiene al ruido generado por los mismos estudiantes (38\%) y por último el ruido generado por el tráfico vehicular (7 \%).

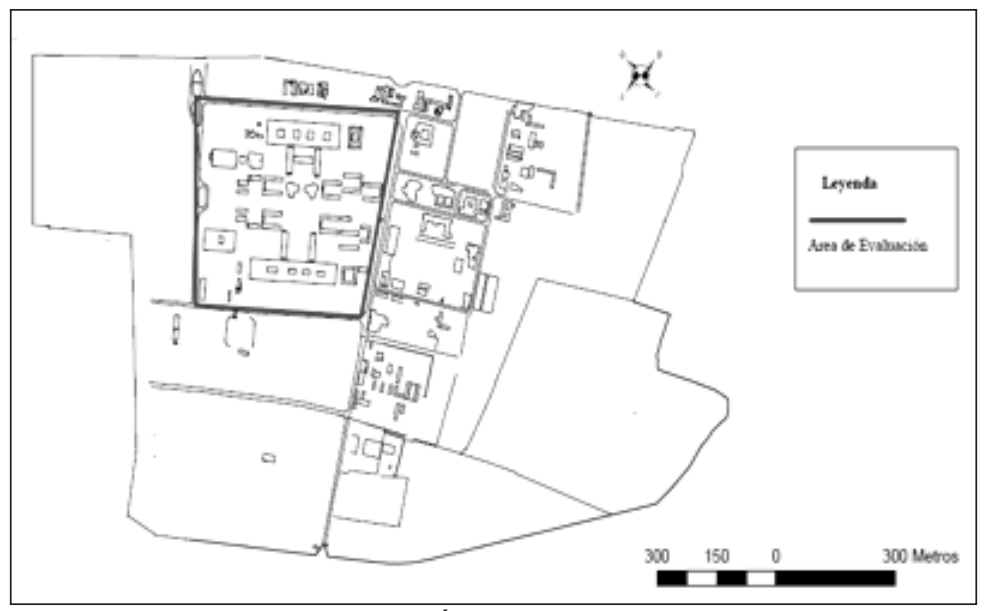

Figura 1. Área de evaluación 


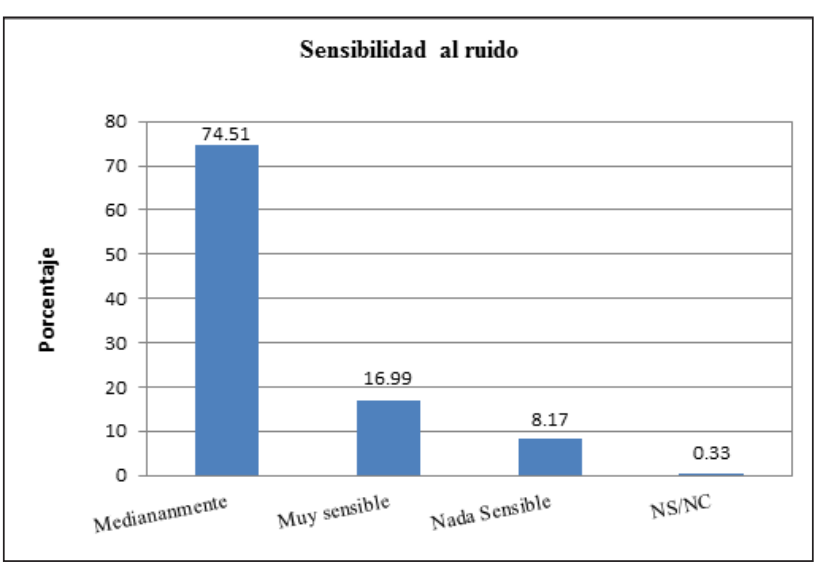

Figura 2. Sensibilidad de los estudiantes de la UNALM al ruido

Respecto a los horarios en los cuales existen mayores niveles de ruido, como se observa en la Fig. 3, el 69 $\%$ afirma que durante los cambios de horario se genera mayor ruido; mientras que el $14 \%$ sostiene que en las mañanas perciben mayores niveles de ruido, el $6 \%$ afirma que existen mayor niveles de ruido por la tarde, el $2 \%$ considera que el campus presenta un ruido continuo durante todo el día y el $8 \%$ considera que el campus no es ruidoso en ningún horario.

Para el $37 \%$ de la población encuestada, el módulo que presenta mayores niveles de ruido son las aulas azules, mientras que un $12 \%$ afirma no distinguir qué módulo le parece más ruidoso y un $16 \%$ afirma que todos los módulos tienen los mismos niveles de ruido.

Respecto al ruido en el interior de las aulas, un $46 \%$ considera que es audible pero no molesta ni interfiere con el proceso de aprendizaje, mientras que el $41 \%$

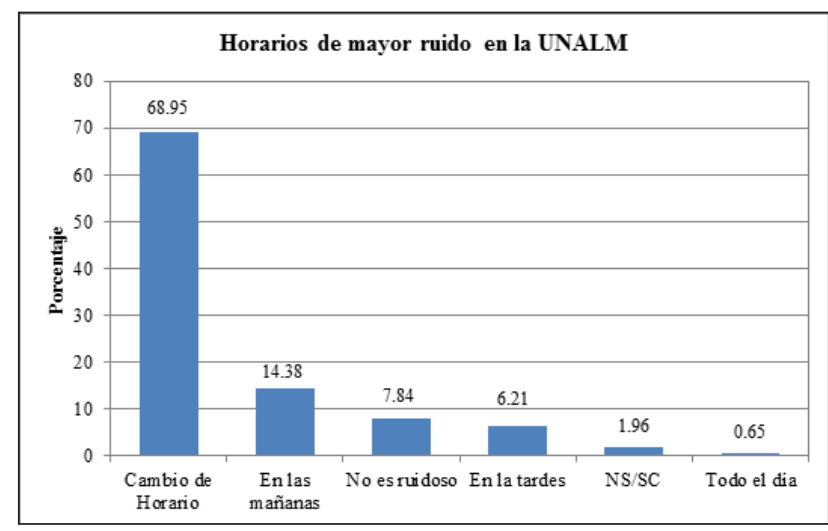

Figura 3. Horario de mayor ruido en la UNALM

percibe el ruido interno como molesto, el $3 \%$ afirma que el ruido interno es medianamente molesto y, por último, un $10 \%$ no percibe ningún ruido molesto dentro de las aulas; la mayoría de los estudiantes que consideran que el ruido al interior de las aulas es molesto, afirma que este es generado por los mismos alumnos. Sin embargo, la mayoría de los estudiantes encuestados no tiene problemas para oír al profesor dentro del aula.

Respecto de las características de la población, no se encontró una variación significativa de percepción entre personas que hayan crecido en Lima y en provincia. En cuanto a la diferencia de percepción del ruido entre géneros, se observó que el género femenino percibe mayor molestia de ruido ambiental, siendo esta diferencia de percepción no significativa, como se observa en la Fig. 4.

Ruido exterior e interior en las áreas académicas de la UNALM.

Respecto al ruido ambiental en las áreas académicas de la UNALM, como se muestra en la Fig. 5, los valores más bajos se registraron en la parte central del área evaluada, correspondiente a las aulas de los módulos rojo y anaranjado, donde se registraron valores de ruido entre 52 y $53 \mathrm{~dB}$; el área correspondiente a las aulas de los módulos

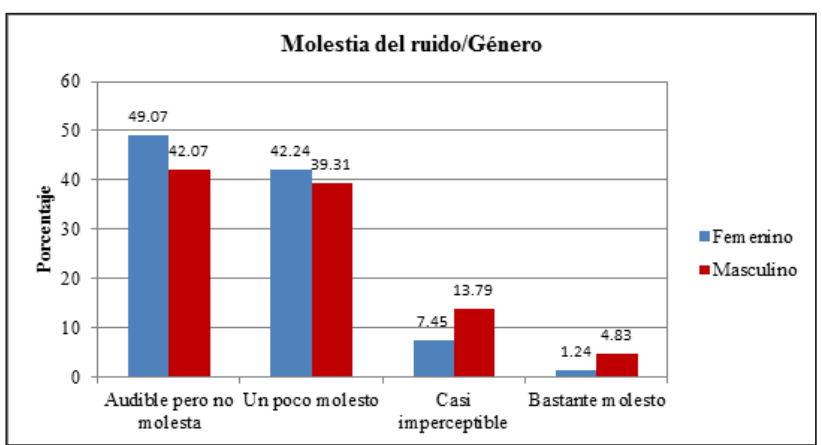

Figura 4. Grado de molestia del ruido respecto al género

amarillo, verde, azul y los auditorios, presenta valores de ruido entre $53 \mathrm{~dB}$ y $55 \mathrm{~dB}$; en las zonas aledañas a las vías principales de la UNALM se registraron niveles de ruido hasta $64 \mathrm{~dB}$. Además, se observó que el área donde se encuentra la BAN presentó niveles de ruido entre $60 \mathrm{~dB}$ y $62 \mathrm{~dB}$ y el área correspondiente al comedor universitario, entre $56 \mathrm{~dB}$ y $58 \mathrm{~dB}$.

\section{Percepción del ruido en la Biblioteca Agrícola Nacional}

En la Fig. 6 se muestra el mapa de ruido interior del primer piso de la BAN en el cual se observa que los mayores niveles de ruido se registraron en el área cercana a las puertas de acceso, ya que por este espacio existe mayor tránsito de estudiantes y por la ubicación de la sala de ciencias, que es una de las áreas más concurridas de la BAN. En la parte donde se encuentran los módulos de lectura se registraron los menores valores de ruido, sin embargo, presenta valores que exceden en casi $20 \mathrm{~dB}$ las recomendaciones de la OMS.

En el segundo piso de la BAN los valores más elevados se registraron en la parte correspondiente a la sala de agricultura, $58 \mathrm{~dB}$ a $60 \mathrm{~dB}$ aproximadamente, debido a que esta zona se encuentra junto a las escaleras de acceso y el diseño de las mesas de estudio grupal propicia la conversación y murmullo entre los estudiantes; así también se puede observar que a medida que se llega hacia la Sala Perú, los niveles de ruido disminuyen hasta llegar a $51 \mathrm{~dB}$ aproximadamente. 


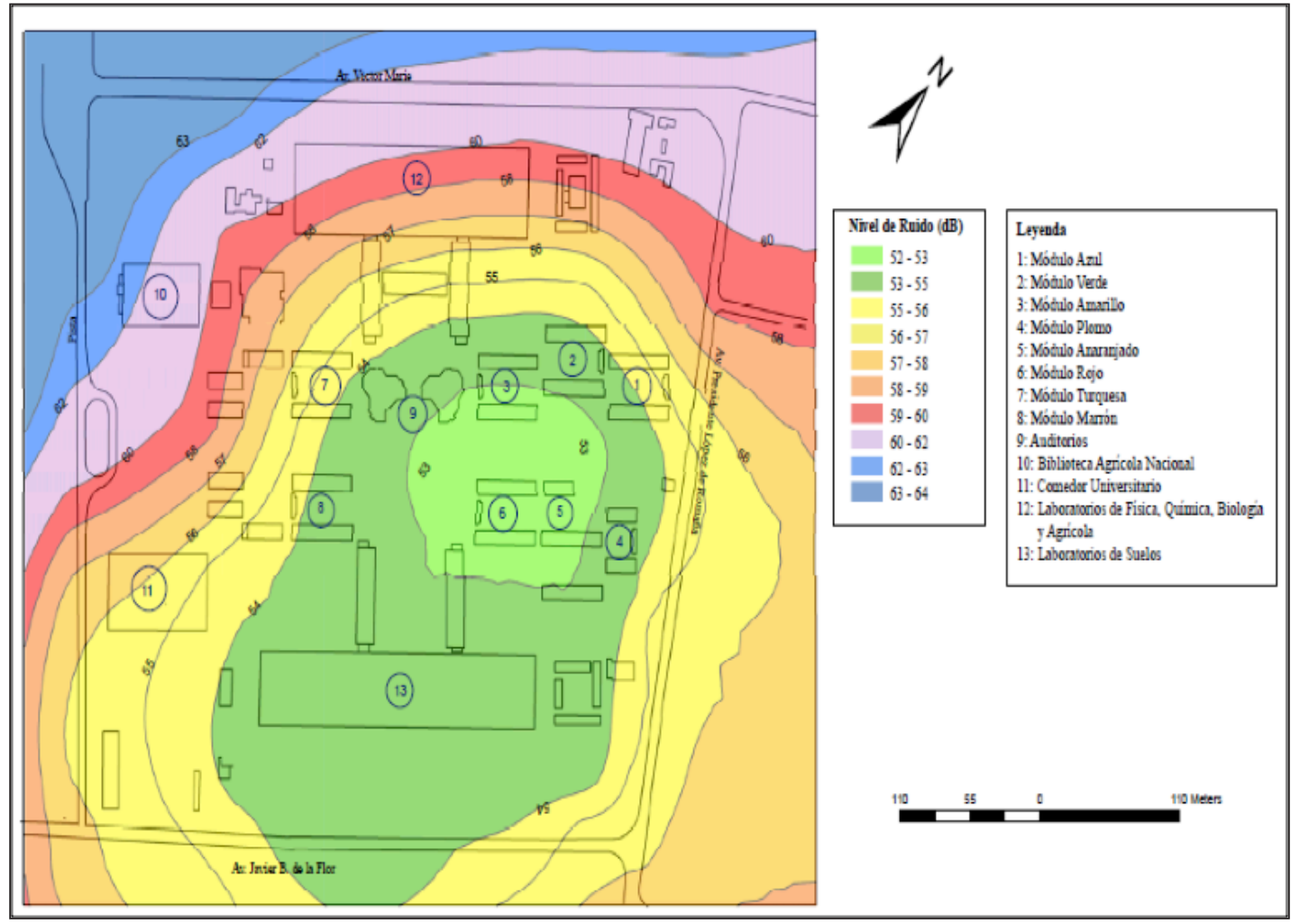

Figura 5. Mapa acústico de las áreas académicas de la UNALM

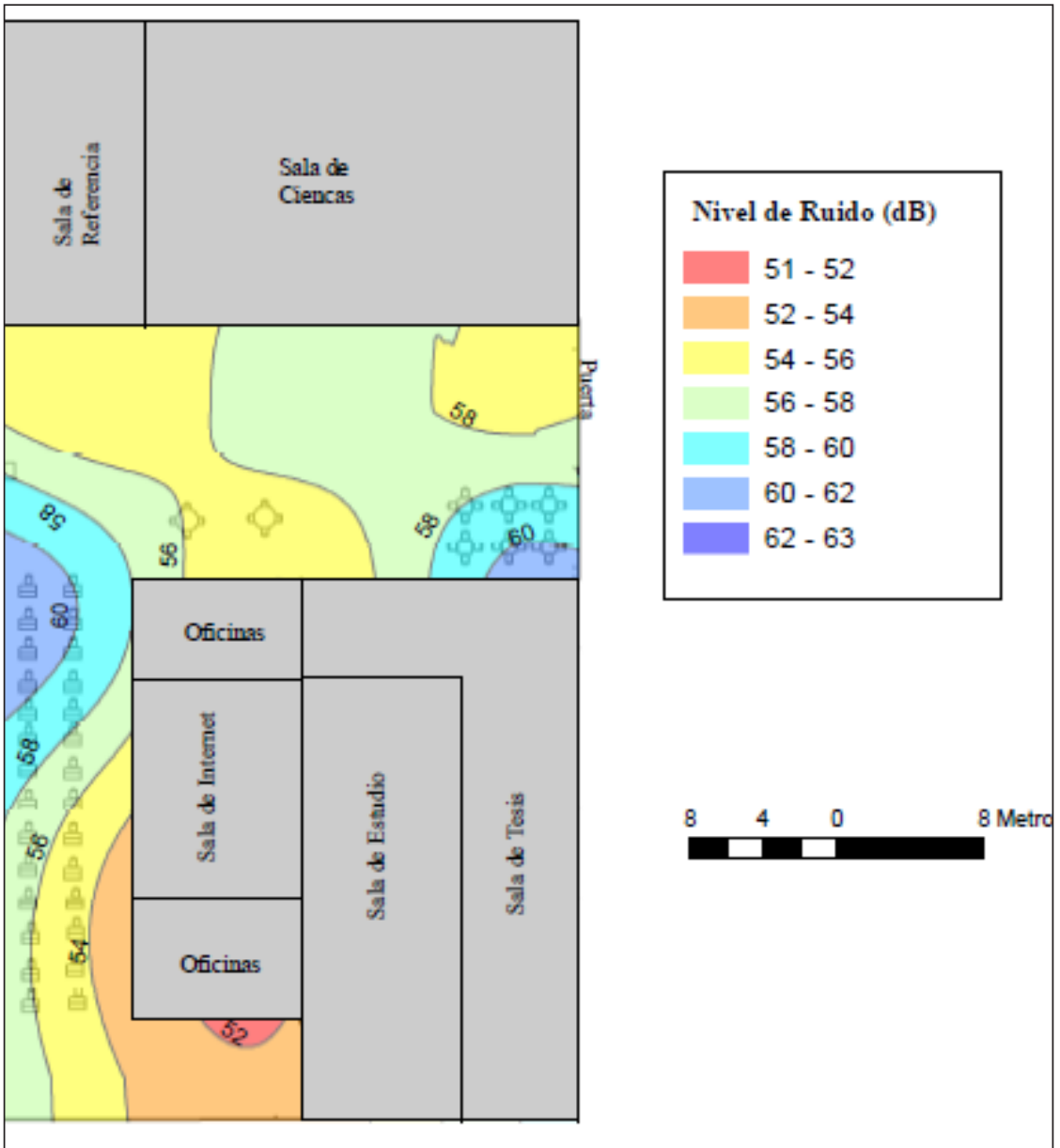

Figura 6. Mapa de ruido interior en el primer piso de la BAN 
El $64 \%$ de las personas que asisten a la BAN no tiene dificultad de concentración dentro de esta, como la mayoría de los estudiantes que asisten a la BAN con una frecuencia de una a tres veces por semana; mientras el $13 \%$ lo hace a diario. Para poder determinar la molestia que el ruido interior de la BAN ejerce sobre los estudiantes que asisten regularmente a este lugar, es necesario saber el tiempo que permanecen dentro de ella (Del Sante, 2011). Al respecto, el $33 \%$ de los estudiantes encuestados permanece dentro de la BAN una hora a más tiempo, mientras que un $25 \%$ afirma que solo permanece un máximo de veinte minutos, el $21 \%$ un máximo de 40 minutos y un $15 \%$ un máximo de una hora; siendo el horario de la mañana, especialmente a partir de las 11:00 horas, cuando se da el mayor ruido en la BAN.

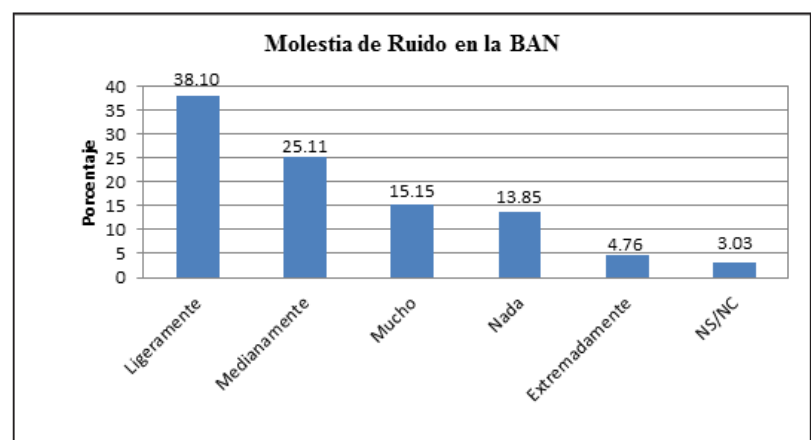

Figura 7. Grado de molestia del ruido para los estudiantes que asisten a la BAN
En cuanto a la molestia del ruido dentro de la Biblioteca, la mayoría de los estudiantes afirma que molesta ligeramente, en $38 \%$, sin que altere las actividades que realizan, una menor proporción de los estudiantes afirma que molesta medianamente, en $25 \%$, como se observa en la Fig. 7, esto a pesar de los altos niveles de ruido registrados en las diferentes salas de estudio (Fig. 6). También se pudo confirmar que hay un $19 \%$ que pocas veces se puede concentrar dentro de la BAN y un $4 \%$ que casi nunca puede concentrarse. La mayoría de los estudiantes que constantemente acuden a la BAN afirman no tener problemas de comunicación dentro de esta; a pesar de esto sí consideran necesario un mejor diseño acústico de la BAN para poder mejorar sus características acústicas.

Percepción del ruido ambiental en el comedor universitario.

El mapa de ruido interior del comedor universitario se muestra en la Fig. 8, la variación de los niveles de ruido es de $15 \mathrm{~dB}$ aproximadamente, $(65-81 \mathrm{~dB})$, los menores valores de ruido se ubican en el lado izquierdo del comedor universitario y se va incrementando hacia la parte central y la salida de este; la gradiente de variación es mayor hacia el lado izquierdo también; los valores de ruido más altos se registran en el área cercana al depósito de charolas, $80 \mathrm{~dB}$ aproximadamente, produciendo enmascaramiento de la voz y el acto de conversar se torna dificultoso (López, 1997).

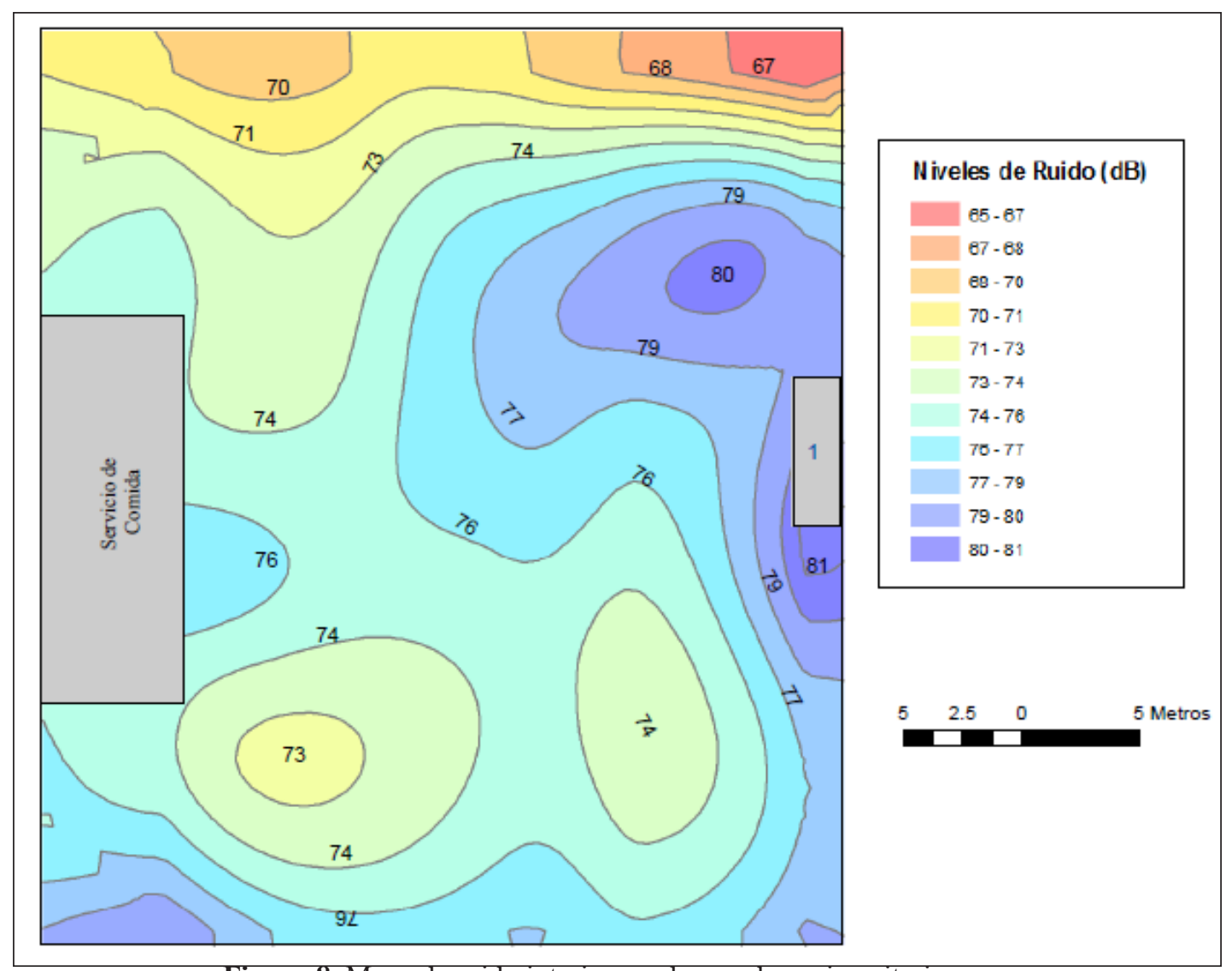

Figura 8. Mapa de ruido interior en el comedor universitario 
La OMS no recomienda un valor máximo permisible para comedores o afines, pero en algunos países europeos y latinos se ha adoptado un intervalo permisible entre 40 - 60 dB (Cámara de Comercio de Bogotá, 2004), los valores registrados dentro del comedor universitario superan excesivamente esta recomendación.

El $50 \%$ de los estudiantes encuestados asiste esporádicamente al comedor universitario, mientras que un $23 \%$ lo realiza diariamente, un $20 \%$ lo hace tres veces por semana y, por último, un $10 \%$ lo hace una vez por semana; según algunas opiniones recibidas durante la realización de encuestas, los estudiantes manifestaron que su frecuencia de asistencia se debe a la modalidad de comprar los tickets que se ha implementado actualmente.

El horario de mayor ruido, en el área del comedor universitario, es de las 13:00 a las 14:00 horas, ya que en este intervalo la mayoría de alumnos almuerza; por lo tanto en ese periodo el comedor universitario se encuentra lleno.

El $46 \%$ de los estudiantes permanece un máximo de 20 minutos dentro del comedor universitario, el $38 \%$ permanece entre 20 y 40 minutos, el $9 \%$ entre 40 minutos y una hora y el $7 \%$ más de una hora. Esta información pertenece únicamente al horario de almuerzo.

Dentro del comedor universitario, el ruido más molesto es el generado por las charolas cuando son depositadas en la parte inferior del recinto, cómo se muestra en la Fig. 9, el $28 \%$ de los estudiantes sienten mucha molestia por este ruido, al $21 \%$ le molesta ligeramente y medianamente, al $15 \%$ extremadamente y al $14 \%$ no le molesta nada.

El $66 \%$ de los estudiantes que asisten al comedor tienen que alzar la voz para poder comunicarse, mientras que el $26 \%$ necesita casi gritar para lograr ser escuchado, por último el $8 \%$ afirma que sí puede conversar perfectamente, como se muestra en la Fig. 10. Esta

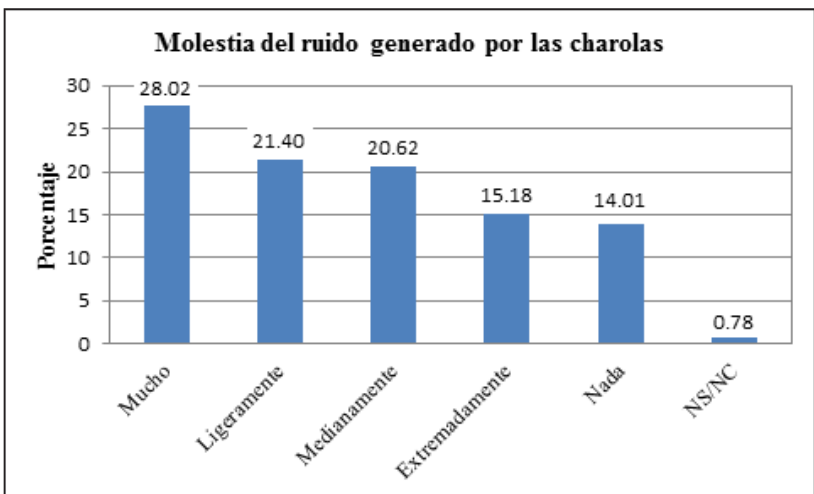

Figura 9. Molestia del ruido generado por las charolas

situación depende del horario en el que los estudiantes asisten al comedor universitario, ya que si lo hacen antes de las 13 horas los niveles de ruido son menores y es posible que la comunicación oral se realice de forma más fluida. El 90 \% sugieren que es necesario un mejor diseño acústico para este ambiente, ya que los altos niveles de ruido pueden generar diversas descompensaciones en el cuerpo humano como estrés y mal humor (Basrur, 2000).

De acuerdo con López y Herranz (2000), las personas sensibles al ruido sentirán mayor molestia por éste, sin embargo a pesar de que el ruido ambiental en las áreas académicas de la UNALM supera el valor recomendado por la OMS para lugares de estudio, los estudiantes

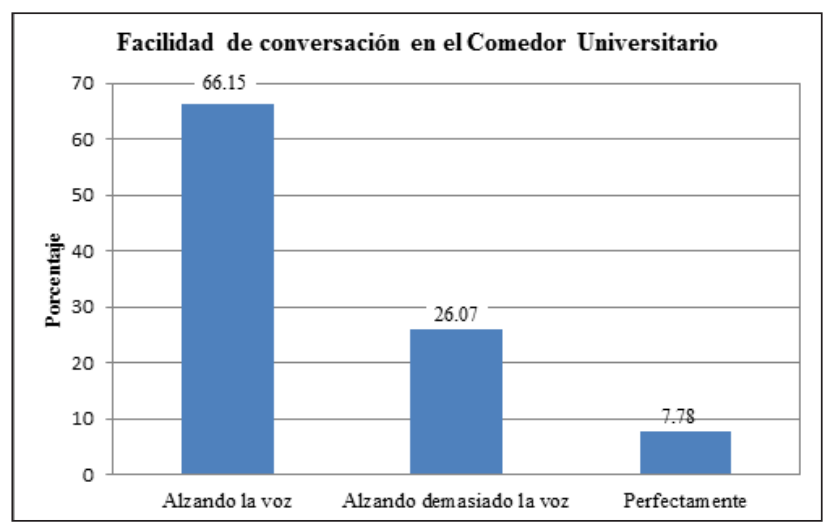

Figura 10. Facilidad de conversación dentro del comedor universitario

de la UNALM no perciben que este ruido sea molesto ni que interfiera de forma negativa en el desarrollo de sus actividades académicas, esto puede deberse a la adaptación a elevados niveles de ruido (Vargas, 1994).

El nivel de molestia también depende de la naturaleza del ruido y de la actividad que se está realizando, mientras los estudiantes no se encuentren realizando actividades académicas es probable que no perciban molestia por ruido (Ordaz, Maqueda, Asunsolo, Silva, Gamo, Cortes y Bermejo, 2009); además, si el ruido actúa como elemento informativo y enriquecedor del medio donde es percibido, la respuesta de la persona ante este es positiva (Aragonés y Amérigo, 2002).

No se encontró una tendencia en la percepción del ruido respecto al lugar de procedencia, ya que según Del Sante (2011) los pobladores que provienen de ciudades menos desarrolladas son más sensibles a los problemas ambientales, esto puede ser causa de que actualmente las pequeñas ciudades están teniendo un crecimiento acelerado y por lo tanto vienen enfrentando problemas de contaminación ambiental, entre los que se encuentra la contaminación acústica.

Según Hunter, Susie y Wayne (2007) el género femenino es más sensible a los problemas ambientales, sin embargo, en el presente estudio no se encontró una diferencia significativa entre el género masculino y femenino sobre la percepción de ruido; según los autores citados, esta situación era resultado del tiempo que las mujeres invertían en los quehaceres domésticos, tendencia que en la actualidad no es muy frecuente.

El ruido de las áreas académicas de la UNALM va aumentando hacia la zona periférica debido a que la parte central se encuentra más protegida de algunas fuentes generadoras de ruido, como vehículos a motor (Posada, 
Arroyave y Fernández, 2009). El módulo azul presenta niveles de ruido más elevados que los otros módulos, esto se debe a su cercanía con las vías de circulación de vehículos a motor dentro del universidad, además de ser el único módulo que posee un segundo nivel (piso), por lo que el tránsito de personas es mayor y el material de las escaleras propicia el incremento de los niveles de ruido.

En el comedor universitario los excesivos niveles de ruido generan mucha molestia ya que se produce un enmascaramiento de la voz (López, 1997), interfiriendo con la comunicación oral, generando que las personas tengan que alzar demasiado la voz, lo cual propicia que el ruido ya existente se eleve.

Las personas que asisten con regularidad a la BAN muestran cierta molestia generada por el ruido, ya que se pierde la concentración fácilmente, generando un estado de estrés y fastidio en la persona. La mayor fuente generadora de ruido en este espacio son los alumnos, como afirman Estrada-Rodríguez y Méndez (2010); el nivel de ruido en la BAN genera bastante malestar ya que interfiere con las actividades del lugar y representa un estímulo para la distracción (Álvarez, 2006).

Según la OMS, en la Guía para el Ruido (1999) los valores recomendados de ruido interior para salas de estudio son de $35 \mathrm{~dB}$ y en la normativa de varios países, tanto europeos como latinos, los valores máximos para estos ambientes son de 35-45 dB (Cámara de Comercio de Bogotá, 2004); en el caso de la Biblioteca Agrícola Nacional, no se cumple la recomendación de la OMS ya que el ruido interior supera los $50 \mathrm{~dB}$ en todo momento, excediendo en $20 \mathrm{~dB}$ aproximadamente los valores recomendados.

Las áreas adyacentes a la BAN y al comedor universitario presentan niveles elevados de ruido, debido a que en estas áreas existe una alta concentración de estudiantes en horas determinadas; además, en el momento de la evaluación se encontraba en funcionamiento un cafetín al lado de la BAN.

\section{Conclusiones}

Los niveles de ruido ambiental de las áreas académicas de la Universidad Nacional Agraria La Molina exceden el nivel establecido para horario diurno por los Estándares Nacionales de Calidad Ambiental para Ruido - ENCA (DS. N085-2003); los módulos azul y turquesa son las aulas que presentan mayores niveles de ruido, mientras que los módulos anaranjado y plomo se presentan como los menos ruidosos.

Dentro de la Biblioteca Agrícola Nacional, la mayoría de los asistentes percibe cierto grado de molestia a causa del ruido interior, a pesar de esto la mayoría de los estudiantes afirma poder concentrarse dentro de la BAN sin mayor dificultad; pero sostienen que es necesario un mejor diseño acústico del lugar. El primer y segundo nivel de la biblioteca presentan niveles de ruido interior que superan el valor recomendado por la Guía para el
Ruido (OMS, 1999); siendo estas áreas no aptas para las actividades de estudio.

En el comedor universitario, los mayores niveles de ruido son percibidos entre las 13:00 y las 14:00 horas; el ruido que más molestia causa a los asistentes es el generado por las charolas, al momento que son depositadas, además del murmullo de las personas; en opinión de los estudiantes, esta zona también requiere de un mejor diseño acústico.

\section{Literatura citada}

Álvarez, G. 2006. Diagnóstico y Propuestas de Gestión de los Impactos Ambientales Producidos por las Estaciones Base Radioeléctricas en el Distrito de San Martín de Porres. (Tesis de maestría). Universidad Nacional Agraria La Molina. Lima, Perú.

Arellano, A. 2008. Distribución del Ruido Ambiental en el Campus de la Universidad Nacional Agraria La Molina en el periodo Enero - Marzo 2007. (Tesis de grado). Universidad Nacional Agraria La Molina. Lima, Perú.

Basrur, S. 2000. Health Effect of Noise. Health Promotion and Environmental Protection Office. Toronto: Public Health.

Cámara de Comercio de Bogotá. 2004. Guía Ambiental: Minimización y Control del Ruido Ocupacional y Ambiental Generado en las Mipymes y Establecimientos Comerciales de Esparcimiento Nocturno. Departamento Técnico Administrativo del Medio Ambiente. Disponible en:www.minambiente.gov.co/Puerta/...ds...guias/ RUIDO.pdf

D.S. No 085 - 2003 - PCM. Reglamento de Estándares Nacionales de Calidad Ambiental (ENCA) para Ruido.

De Esteban, A. 2003. Contaminación Acústica y Salud. Revista Observatorio Ambiental, pp. 73-95. Disponible en: revistas.ucm.es/cca/11391987/articulos/ OBMD0303110073A.

Del Sante, S. 2011. Estudio de la Percepción Ambiental de los Pobladores de la Comunidad de San Juan de Óndores Acerca de los Contaminantes Vertidos en el Lago Junín. (Tesis de grado). Universidad Nacional Agraria La Molina. Lima, Perú.

Hunter, L.; Susie, S. y Wayne, T. 2007. Environmental Perceptions of Rural South African Residents: The complex Nature of a Post-Material Concern. Institute of Behavioral Sciences. University of Colorado at Boulder.

López, I. 1997. Medio Ambiente y Salud. Impacto del Ruido. Instituto de Acústica. Papeles del Psicólogo. Disponible en: http://www.papelesdelpsicologo.es/ vernumero.asp? $\mathrm{id}=753$.

López, I.; Carles, J. y Herranz, K. 2000. El Estudio de los Aspectos Perceptivos en la Acústica Ambiental. Revista de Acústica 31(4), 1-5. Disponible en: http:// digital.csic.es/bitstream/10261/6009/1/Psicoacustica_00. pdf

Ordaz, E.; Maqueda, J.; Asunsolo, A.; Silva, A.; Gamo, M.; Cortes, B. y Bermejo, E. 2009. Efecto de 
la Exposición a Ruido en Entornos Laborales sobre la Calidad de Vida y Rendimiento. Med. Segur. Trab. 552 (16): $35-45$.

ORGANIZACION MUNDIAL DE LA SALUD

(OMS). 1999. Guidelines for community noise. Disponible en: http:/www.who.int/docstore/peh/noise/ guidelines2.html

Posada, M.; Arroyave, M. y Fernández, C. 2009. Influencia de la Vegetación en los Niveles de Ruido Urbano. Revista EIA de Ingeniería de Antioquía. Medellín. (12): 79-89.

Santos, E. 2007. Contaminación por Ruido Vehicular en la Avenida Javier Prado. Industrial Data Enero - Junio, 10 (1), 11 - 15. Universidad Nacional Mayor de San Marcos. Lima, Perú. Disponible en: http://revistas.concytec. gob.pe/scielo.php?script=sci_arttext\&pid=S181099932007000100003\&lng=pt\&nrm=iso

Steel, R. y Torrie, J. 1985. Bioestadística: Principios y Procedimientos. (2da. ed.).

Vargas, L. 1994. Sobre el concepto de percepción. Alteridades, 4 (8): 47-53. 